\title{
Development of a complex intervention aimed at reducing the risk of readmission of elderly patients discharged from the emergency department using the intervention mapping protocol
}

\author{
Louise Moeldrup Nielsen ${ }^{1,2^{*}}$ (1) , Thomas Maribo ${ }^{3,4}$, Hans Kirkegaard ${ }^{5}$, Kirsten Shultz Petersen ${ }^{6}$ \\ and Lisa Gregersen Oestergaard ${ }^{1,3,7}$
}

\begin{abstract}
Background: Limitations in performing daily activities and a incoherent discharge are risk factors for readmission of elderly patients after discharge from the emergency department. This paper describes the development and design of a complex intervention whose aim was to reduce the risk of readmission of elderly patients discharged from the emergency department.

Methods: The intervention was described using the Intervention Mapping approach. In step 1, a needs assessment was conducted to analyse causes of readmission. In steps 2 and 3, expected improvements in terms of intervention outcomes, performance objectives and change objectives were specified and linked to selected theory- and evidence-based methods. In step 4, the specific intervention components were developed; and in step 5, an implementation plan was described. Finally, in step 6, a plan for evaluating the effectiveness of the intervention was described. The intervention was informed by input from a literature search, informal interviews and an expert steering group.

Results: A three-phased theory- and evidence-based intervention was developed. The intervention consisted of 1) assessment of performance of daily activities, 2) defining a rehabilitation plan and 3) a follow-up home visit the day after discharge with focus on enhancing the patients' performance of daily activities.
\end{abstract}

Conclusion: The intervention mapping protocol was found to be a useful method to describe and systemize this theory- and evidence-based intervention.

Keywords: Intervention, Functioning, ADL, Emergency department, Acute care, Occupational therapy, ICF

\section{Background}

Readmission to hospital or Emergency Department (ED) is a common and important healthcare problem among elderly patients in many parts of the world [1-3]. In Western countries, up to $20 \%$ of elderly patients admitted to an ED

\footnotetext{
*Correspondence: Imn@via.dk; losnie@rm.dk

${ }^{1}$ Department of Physiotherapy and Occupational Therapy, Aarhus University Hospital, Aarhus, Denmark

${ }^{2}$ Department of Occupational Therapy, VIA University College, Aarhus, Denmark

Full list of author information is available at the end of the article
}

are readmitted during the first 30 days after their discharge [4-6]. These readmissions have considerable consequences for both the elderly patients and society in general. Readmission is associated with an increase in elderly patients' risk of infections, medical complications and limitations in performing daily activities $[7,8]$. Different factors such as age, comorbidity, medication, diagnoses and activity limitations contribute to elderly patients' risk of readmission and mortality [2, 7]. A large proportion of elderly patients admitted to the ED are discharged directly to their home [9]. Transferring the patients' care and rehabilitation at

(C) The Author(s). 2018 Open Access This article is distributed under the terms of the Creative Commons Attribution 4.0 International License (http://creativecommons.org/licenses/by/4.0/), which permits unrestricted use, distribution, and 
discharge from the ED to primary care is a challenge and involves a risk of lost information, which may influence the patients' experiences of the discharge and their further rehabilitation [10].

Interventions that are aimed at preventing readmission in elderly patients discharged from the ED have been previously evaluated, but there is a lack of consensus regarding which initiatives are the most effective [9-15]. A systematic review from 2005, revealed that different home-based interventions improved the elderly patients performance of daily activities after their discharged from the ED [10]. However, despite this, the home-based interventions did not seem to have any effect on the risk of readmission. A systematic review from 2015 evaluated the effect of transitional interventions for elderly patients discharged from the ED [9]. It found no effect on either readmission or mortality. A systematic review from 2012 evaluated the effect of care coordination after ED discharge and concluded that the majority of studies evaluating such initiatives reported that they were effective in reducing readmission in the elderly [13]. However, the evidence on how to prevent readmission of elderly patients discharged from the ED is inconclusive and conflicting and, as several studies have highlighted, quality studies of the effectiveness of transitional interventions for the elderly are needed $[9,10,13]$. Limitations in performing daily activities have been identified as a predictor for readmission and mortality in elderly patients $[1,2,16,17]$. However, to our knowledge, only a few studies have evaluated the effectiveness of enhancing the elderly patients' performance of daily activities in order to prevent their readmission and reduce their mortality [18-20]. None of these interventions was short-term or conducted in an acute hospital setting. We therefore found it relevant to develop and design a short-term intervention that focused on enhancing the elderly patients performance of daily activities and to ensure a coherent discharge from a short-stay unit at the ED.

The purpose of this paper is to describe the development of a complex intervention that is aimed at reducing the risk of readmission of elderly patients discharged from a short-stay unit at the ED.

\section{Methods}

There is growing understanding that the development and design of interventions should be more transparent [21-23]. The description of the present intervention followed the steps of the Intervention Mapping (IM) protocol for developing health promotion programmes [24]. IM provides a methodological, step-by-step procedure in an iterative process. The six steps in the protocol include several tasks that describe the development process. The first two steps involve the description of a needs assessment and the objectives of the intervention.
In step three, theory-and evidence-based methods and strategies are selected which then inform the intervention developed in step four. Steps five and six describe the plan for implementation and evaluation, respectively [24].

\section{Step 1: Logic model of the problem}

A steering group, a project group and a reference group were established with the aim of bringing expertise to the project. A steering group counting 11 members was established with experts from both hospital and primary care. Five of these experts were also part of the project group, including the project leader. The project group was responsible for planning, implementing and evaluating the intervention. A reference group with physiotherapists (PT) and occupational therapists (OT) from the ED contributed with information about the clinical context. In the developing phase, two meetings with the steering group and approximately five meeting with the reference group were conducted. All decisions from those meetings were based on discussion. If any disagreement should occur, the project leader had the final decision.

Then a needs assessment was performed based on findings from the literature, and informal interviews with health professionals from the hospital and primary care were undertaken. The needs assessment was structured using a logic model that defined phase 1) the problem; phase 2) risk factors; phase 3) underlying behavioural and environmental factors that could affect the risk factors; and phase 4) determinants for the behavioural and environmental factors [24]. After conducting the needs assessment, the context for the intervention was described based on input from clinical experts from the steering group, reference group and the literature. Finally, the goals for the intervention were set.

\section{Step 2: Outcomes and objectives}

To outline the goals for the intervention, we identified overall outcomes for behavioural and environmental change after discussions in the project group and the reference group. The overall outcomes were then divided into separate performance objectives that explicitly described what should happen in order to achieve the outcome. The most important internal (relates to the person) and external (relates to the environment) determinants, identified in step 1 , were then combined with the specified performance objectives to formulate change objectives. These change objectives were actions that specified what would change in the determinants as a result of the intervention and were required in order to achieve the performance objectives and the overall outcome. The performance objectives and change objectives were then discussed in the project group before matrices for behavioural and environmental changes were constructed. 


\section{Step 3: Selecting methods and strategies}

A search of the literature was undertaken to identify theoryand evidence-based methods that relate to the change objectives in step 2 and that could influence change in the determinants and outcomes.

First, we searched for tests to assess limitations in elderly patients' performance of daily activities (please see Additional file 1). Tests were selected on the basis that they were performance-based generic tests that were validated for the elderly population, and that were simple to administer in a clinical setting. Next, we searched for studies that examined the effect of interventions that aimed at reducing the risk of readmission (please see Additional file 2). The identified methods from the literature were then linked to the change objectives in the form of practical strategies suitable for implementation in the concrete setting. Decisions about methods and suitable strategies were made in conjunction with the reference group.

\section{Step 4: Developing intervention components}

Key components of the intervention were selected based on the identified criteria of importance, feasibility and resource constraints. The components were described and practical applications for use in the different components were constructed. A description for performing the components in the intervention was developed and component 1 was pretested with a similar population of elderly patients admitted to the ED. The tests in component 1 , identified in step 3 , were pretested in a two weeks period, in order to examine whether the tests were possible to use in an acute setting. The pretest was done by the therapists responsible for delivering the intervention. During the pretest, the therapist received supervision from the project leader in order to ensure that the test was used as described.

\section{Step 5: Implementation plan}

A plan for ensuring the implementation of the intervention was conducted in cooperation with the reference group. Potential problems and barriers associated with implementation of the intervention were discussed with the reference group. Also, a plan for educating health professionals performing the intervention was devised.

\section{Step 6: Evaluation plan}

In step 6, we developed a plan for evaluating the effectiveness of the intervention and for examining the elderly patients' experiences of being discharged from the ED and their return to everyday lives. A protocol was drawn up that described the design of the study, aim, hypothesis, recruitment plan and the methods used to evaluate the intervention. We also conducted a pilot study designed as a randomized controlled trial to test the feasibility of the intervention and to examine how the intervention could be delivered in practice. The pilot study was evaluated by registration of how many patients it was possible to include, how many refused to participate, time used for component 1 and registration of the possibility of referral of rehabilitation plan and follow-up visits.

\section{Results}

The results of the development process are presented following the six steps as described in the method section. Steps 1 to 3 address the development of the intervention, step 4 presents the final intervention and steps 5 to 6 describe the implementation and evaluation plan.

\section{Step 1: Logic model of the problem}

In the needs assessment, we defined the overall problem as high risk of hospital readmission in the elderly after their discharge from an short stay unit at the ED. This is a well-described problem in the literature $[1,7,16,25]$ and is supported by experiences of the health professionals involved in developing the intervention. The outcome of the needs assessment is presented in the logic model in Fig. 1.

Factors associated with the risk of readmission of elderly patients were identified from the literature. Limitations in performing daily activities was chosen as the most significant risk factor, as a large proportion of elderly patients readmitted has limitations in performing daily activities and because it is a factor that is possible to address in an acute setting with a short-time frame. In the elderly, both the perceived illness and hospitalisation involve a risk of limitations in performing daily activities [26-30]. Another important factor associated with risk of readmission was a incoherent discharge [31, 32]. After ranking the risk factors, underlying behavioural and environmental factors and determinants for the two risk factors were identified using the International Classification of Functioning, Disability and Health (ICF) and the Model of Human Occupation (MoHO) [33-35]. The focal points chosen for further development were the behavioural factor 'decreased ability to perform daily activities' and the two environmental factors, 'poor accessibility in the home' and 'poor coordination between hospital and primary care' (see Fig. 1). The internal determinants that influence a person's ability to perform daily activities were identified as decreased skills in performing daily activities. When a person experiences decreased skills, his or her way of performing daily activities may change in relation to efficiency, effort, safety and independence [36]. In addition, the environment can influence the elderly individual's ability to perform daily activities by either enabling or inhibiting performance. Accessibility in the patient's home and in the community was identified as 


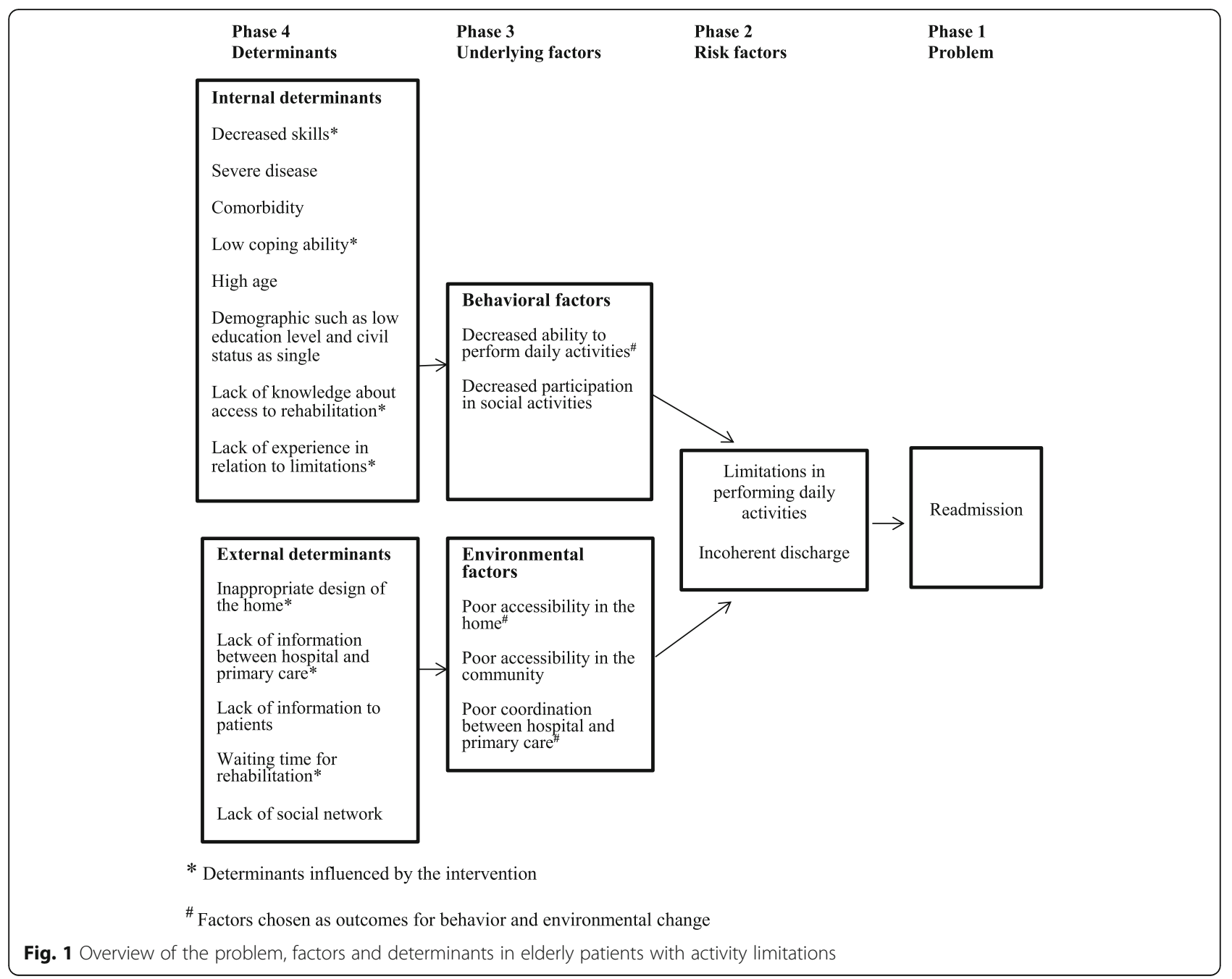

important environmental factors in relation to performing daily activities [35].

On the basis of informal interviews with health professionals, the determinant related to the identified incoherent discharge from the short stay unit at the ED was defined as lack of information exchange between health professionals from the hospital to primary care. Waiting time for rehabilitation and lack of information provided to the patient were also identified as determinants for a incoherent discharge.

Based on the needs assessment, the goals of the intervention were to reduce the risk of readmission by:

- Enhancing the patient's performance of daily activities

- Ensuring a coherent discharge

\section{Step 2: Outcomes and objectives}

Specific outcomes related to behavioural and environmental factors were stated. The outcomes were selected on their basis of considerations regarding their potential to influence readmission, as described in the literature. Furthermore, the outcome had to be both changeable and possible to coordinate in the acute setting. The outcomes were:

- Increase the patient's ability to perform daily activities

- Increased accessibility in the home

- Enhanced coordination between hospital and primary care.

The outcome 'Increasing the patient's ability to perform daily activities' was divided into five performance objectives, and the two outcomes that related to the environment were divided into three performance objectives (see Table 1). Then, the most important and changeable determinants (as identified in step 1) were combined with the specified performance objectives in the form of change objectives in a matrix. The matrix for the behavioural and environmental outcomes is presented in Table 1. 
Table 1 Matrix of performance objectives, change objectives and determinants in elderly patients with limitations in the ability to perform daily activities

\begin{tabular}{|c|c|c|c|c|c|}
\hline \multirow[t]{2}{*}{ Time/setting } & \multirow[t]{2}{*}{$\begin{array}{l}\text { Performance objectives, } \\
\text { patients related }\end{array}$} & \multicolumn{4}{|c|}{$\begin{array}{c}\text { Internal } \\
\text { determinants }\end{array}$} \\
\hline & & Performance skills & Coping ability & $\begin{array}{l}\text { Lack of knowledge } \\
\text { about access } \\
\text { to rehabilitation }\end{array}$ & $\begin{array}{l}\text { Lack of experience } \\
\text { in relation to } \\
\text { current situation }\end{array}$ \\
\hline Day 0/ At the ED & $\begin{array}{l}\text { Decide to participate } \\
\text { in assessment of } \\
\text { activity limitations }\end{array}$ & & & $\begin{array}{l}\text { Receive relevant } \\
\text { information about } \\
\text { the assessment }\end{array}$ & $\begin{array}{l}\text { Recognize that } \\
\text { the ability to } \\
\text { perform activities } \\
\text { have changed } \\
\text { due to illness }\end{array}$ \\
\hline Day 0/ At the ED & $\begin{array}{l}\text { Participate in performance- } \\
\text { based assessment }\end{array}$ & $\begin{array}{l}\text { Agree to be } \\
\text { assessed in relation } \\
\text { to perform activities }\end{array}$ & & & $\begin{array}{l}\text { Experience } \\
\text { possible change } \\
\text { in performance } \\
\text { of daily activities }\end{array}$ \\
\hline Day 0/ At the ED & $\begin{array}{l}\text { Decide to participate in } \\
\text { further rehabilitation }\end{array}$ & Agree to participate & & $\begin{array}{l}\text { Achieve and consider } \\
\text { information about } \\
\text { opportunities for } \\
\text { further rehabilitation }\end{array}$ & $\begin{array}{l}\text { Recognize that } \\
\text { the ability to } \\
\text { perform daily } \\
\text { activities have } \\
\text { changed }\end{array}$ \\
\hline $\begin{array}{l}\text { Day } 1 \text { and after/ } \\
\text { Patient home }\end{array}$ & Perform the training & $\begin{array}{l}\text { Train to perform } \\
\text { activities in a } \\
\text { different way } \\
\text { Train motor and } \\
\text { process skills }\end{array}$ & $\begin{array}{l}\text { Train in how to ask for } \\
\text { assistance and/or help } \\
\text { Consider information } \\
\text { about possible strategies }\end{array}$ & & \\
\hline \multirow[t]{2}{*}{ Time/setting } & $\begin{array}{l}\text { Performance objectives, } \\
\text { staff related }\end{array}$ & \multicolumn{4}{|c|}{ External determinants } \\
\hline & & $\begin{array}{l}\text { Lack of information } \\
\text { between hospital } \\
\text { and primary care }\end{array}$ & $\begin{array}{l}\text { Waiting time for } \\
\text { rehabilitation } \\
\text { after discharge }\end{array}$ & $\begin{array}{l}\text { Inappropriate design of the } \\
\text { patient's home }\end{array}$ & \\
\hline Day 0/ At the ED & $\begin{array}{l}\text { Inform primary care about } \\
\text { patient being discharged } \\
\text { and plans for further } \\
\text { rehabilitation }\end{array}$ & $\begin{array}{l}\text { OT prescribe } \\
\text { rehabilitation plan } \\
\text { OT at the ED contacts } \\
\text { therapists from } \\
\text { primary care }\end{array}$ & $\begin{array}{l}\text { Fast referral of the } \\
\text { patient }\end{array}$ & & \\
\hline Day 0/ At the ED & $\begin{array}{l}\text { Change visitation } \\
\text { procedure for patients } \\
\text { referral }\end{array}$ & & $\begin{array}{l}\text { Make directly contact } \\
\text { to therapists from } \\
\text { primary care }\end{array}$ & & \\
\hline Day $1 /$ Patient home & $\begin{array}{l}\text { Access accessibility } \\
\text { in the patients home }\end{array}$ & & & $\begin{array}{l}\text { Screen the patients } \\
\text { home in relation to } \\
\text { safety risk when } \\
\text { performing daily activities }\end{array}$ & \\
\hline Day 1 / Patient home & $\begin{array}{l}\text { Make minor necessary } \\
\text { changes in patients home }\end{array}$ & & & $\begin{array}{l}\text { Remove carpets } \\
\text { Arrange furniture }\end{array}$ & \\
\hline
\end{tabular}

\section{Step 3: Selecting methods and strategies}

In order to address the determinants and performance objectives specified in step 2, suitable theoretical and evidence-based methods were identified in the literature. Based on our search of the literature, we found the most frequently reported approaches used to increase the performance of daily activities to be skill development, task and environmental modification, and the use of assisted devices [20, 37, 38]. We found sparse evidence on the following environmental outcomes: safety and prevention, use of adaptive equipment, environmental modification and assisted devices [20,37]. There seems to be evidence that skills training leads to increased ability to perform daily activities [38, 39]. The evidence-based methods were then supplemented with theoretically derived methods and practical strategies from the Behaviour Change Techniques taxonomy [40] and MoHO [35]. Table 2 shows the identified methods and practical strategies applied for each determinant that related to each performance objective.

\section{Step 4: Developing intervention components}

The practical strategies were combined to produce the intervention which consisted of three different components (Fig. 2). 
Table 2 Determinants, methods and practical applications used to realize change objective in elderly patients with activity limitations discharge from the emergency department

\begin{tabular}{|c|c|c|}
\hline Determinant $^{a}$ & Methods ${ }^{b}$ & Practical applications/Strategies ${ }^{c}$ \\
\hline \multirow[t]{6}{*}{ Performance skills } & Assessment & $\begin{array}{l}\text { OT and PT at the ED use performance-based tests to assess the patients ability } \\
\text { to perform daily activities }\end{array}$ \\
\hline & Information & OT at the ED gives oral and written information about test result \\
\hline & Tailoring & OT at the ED match the further intervention to the patients need of rehabilitation \\
\hline & Acquisitional approach & $\begin{array}{l}\text { Skills training with the OT after discharge using graduated daily activities until the } \\
\text { goal of activity is achieved }\end{array}$ \\
\hline & Restorative approach & $\begin{array}{l}\text { Skills training with the OT after discharge using graduated daily activities until } \\
\text { the goal of body function is achieved }\end{array}$ \\
\hline & Adaptive approach & $\begin{array}{l}\text { OT from primary care teach alternative or compensatory strategies and teach } \\
\text { in use of assistive devises after discharge }\end{array}$ \\
\hline Coping ability & Feedback & $\begin{array}{l}\text { OT gives the patient information regarding the extent to which they accomplish } \\
\text { learning and performance }\end{array}$ \\
\hline \multirow[t]{2}{*}{$\begin{array}{l}\text { Knowledge about access } \\
\text { to rehabilitation }\end{array}$} & Information & $\begin{array}{l}\text { The patient receive oral and written information about opportunities } \\
\text { from the OT at the ED }\end{array}$ \\
\hline & Consulting & OT from the ED advise the patient about opportunities \\
\hline $\begin{array}{l}\text { Lack of experience in relation } \\
\text { to new situation }\end{array}$ & Direct experience & $\begin{array}{l}\text { The patient performs daily activities both at the ED and at the home } \\
\text { visit the day after discharge }\end{array}$ \\
\hline $\begin{array}{l}\text { Inappropriate design of } \\
\text { the home }\end{array}$ & Adaptive approach & $\begin{array}{l}\text { The OT from the ED advices on minor home modification at the home } \\
\text { visit the day after discharge }\end{array}$ \\
\hline \multirow{2}{*}{$\begin{array}{l}\text { Lack of information between } \\
\text { hospital and primary care }\end{array}$} & Information & OT uses results from the tests in the patients rehabilitation plan \\
\hline & Intergroup contact & $\begin{array}{l}\text { Telephone meetings between OT/PT's at the ED and form primary care } \\
\text { to coordinated discharge and further rehabilitation }\end{array}$ \\
\hline \multirow[t]{2}{*}{ Waiting time for rehabilitation } & Change visitation process & The project leader conducts meetings with chief of rehabilitation from primary care \\
\hline & $\begin{array}{l}\text { Start training immediately } \\
\text { after discharge }\end{array}$ & The OT from the ED conducts home visit with training the day after discharge \\
\hline
\end{tabular}

${ }^{\text {a }}$ Determinants identified in the needs assessment step 1

${ }^{\mathrm{b}}$ Methods identified in the literature that could influence change in the determinants

'Practical applications/strategies describes how the method practically could be delivered

Component 1 involved an assessment, lasting up to two hours, of individual patients' performance of daily activities at the ED. Three performance-based measures Timed-Up and Go, 30s-Chair Stand Test and the Assessment of Motor and Process Skills were selected as the test battery and performed by OTs and PTs [41-45].

A rehabilitation plan was then conducted for patients with identified activity limitations in component 2 . After discharge, the patient's rehabilitation plan was used as a referral to further rehabilitation in primary care. Primary care practitioners were informed about the patient's discharge, and referral of the patient to further rehabilitation was carried out the same day with the aim of starting rehabilitation immediately after discharge.

In component 3 , the OT who assessed the patient and defined the rehabilitation plan visited the patient at home the day after discharge in order to enhance the patient's independence in performing daily activities. The OT used an adaptive and/or an acquisitional approach [36]. The OT screened the home for safety risks and factors that could potentially limit the individual's performance of daily activities. If limitations and/or risk factors for safety were identified, the OT provided advice on modifications of the home environment. Moreover, the OT encouraged the patient to perform daily activities and provided direct training on how a specific activity could be performed differently to enable the patient to perform the activity. To ensure standardised procedures in the intervention, a checklist was developed to guide the OT at the home visit. Additionally, these visits aimed to ensure a coherent post-discharge period.

\section{Step 5: Implementation plan}

As part of the developed plan for implementation, the PTs and OTs delivering the intervention participated in a one-day training course that introduced the components in the intervention. After this introduction, the therapists received supervision and feedback on how they delivered the intervention during the first weeks of implementation. During the recruitment period, weekly meetings were organised between the participating staff and the project leader with the aim of discussing and solving potential problems. Meetings between healthcare managers from primary care and the project leader were held to discuss implementation of the rehabilitation plans. At each primary care unit (eight) in the catchment 


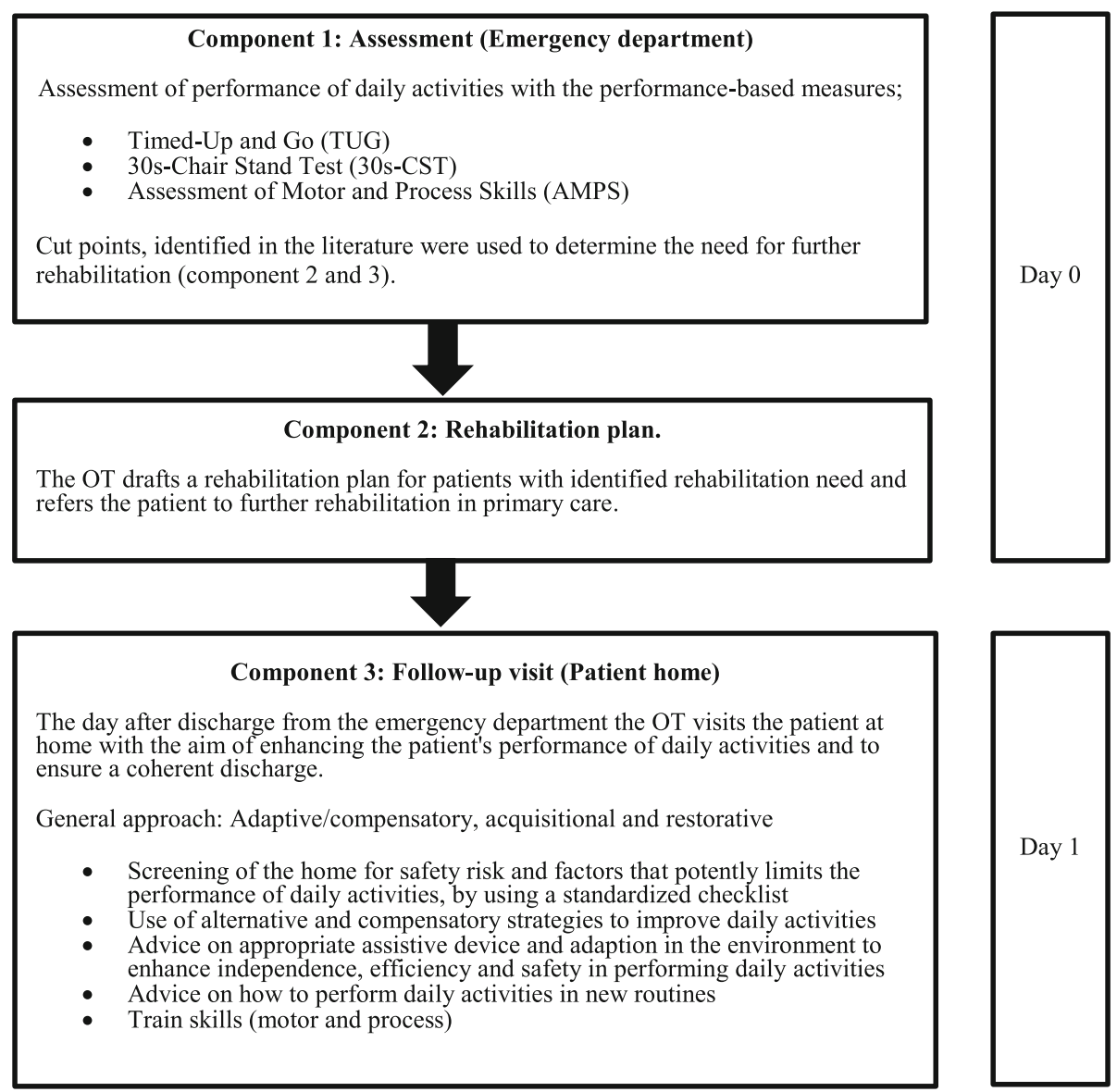

Fig. 2 Overview of the intervention components in the Elderly Activity Performance Intervention

areas, a contact person (PT or OT) was appointed to the study in order to ensure early initiation of the rehabilitation plan. These contact people participated in a day course that introduced them to the components of the intervention. Meetings with PTs and OTs from primary care who refer patients to further rehabilitation were held with the aim of discussing how quick referrals could be conducted to minimise waiting time for patients.

\section{Step 6: Evaluation plan}

An evaluation of the intervention will be conducted in order to evaluate the following aims:

1. The effectiveness of the intervention with respect to readmission of elderly patients discharged from the ED

2. The cost-effectiveness of the intervention

3. Change in activity performance for patients in the intervention group

4. The elderly patient's experience of being discharged and returning to everyday life after an acute admission
To test the acceptability of the intervention, recruitment and randomization procedures before conducting a large scale study, we made a pilot study designed as a randomised controlled trial. The study included 52 patients allocated to the intervention $(n=24)$ and the usual practice group $(n=28)$. The pilot study revealed that it was difficult to include patients as $67 \%$ of the eligible patients refused to participate. One of the main reasons for patients to refuse was that they, becauce of the randomisation procedures, had to agree to participate before knowing which group they would be assigned to. The evaluation from the pilot study also revealed that the intervention was feasible to deliver and the design of the intervention therefore was not changed.

A quasi-experimental study with an intervention and a control group will be conducted (Clinicaltrial.gov, NCT02078466). Inclusion criteria are the following: age $65+$, residency in a larger city in Denmark, admission at a short-stay unit at the ED at a university hospital for medical reasons with the expectation of being directly discharged home. Exclusion criteria are patients with terminal illness, dementia, not speaking Danish or transferral to another hospital department. Due to limited 
resources and time, it will be possible to include and allocate up to two patients in the intervention group per day, Monday - Friday. Each weekday at 8.00 am, a research therapist will review all patients admitted in the last $24 \mathrm{~h}$ and screened for eligibility. If more than two patients are eligible, allocation will based on the date of birth so that patients born closest to the first day of a month (e.g. March 1st) will be allocated to the intervention group. Patients not included in the intervention group will be allocated to usual practice group. In addition, patients admitted after $8.00 \mathrm{am}$ meeting the inclusion criteria and discharged out of hours (afternoons and evenings) will be allocated to the usual practice group.

The effectiveness of the intervention (aim 1) will be analysed by comparing the intervention group with the usual practice group in relation to readmission, mortality and contacts to general practitioners, emergency physician and the ED. The primary outcome is allcause readmission within 26 weeks registered in the National Patient Register. A follow-up time of 26 weeks is chosen as it is considered appropriate for enhancing elderly patients performance of daily activities [1]. The secondary outcomes are mortality and number of contacts to general practitioners, an emergency physician and the ED within 26 weeks. Readmission within 30 days is also measured as a secondary outcome. Based on the literature, we assume that the intervention can reduce the risk of readmission within 26 weeks from 37 to $21 \%$ [46]. Power analysis revealed that the sample size should consist of 152 patients in each group, assuming that $10 \%$ of the participants are lost to follow-up. This implies that a total of 304 patients will be needed to detect a risk difference of 16 percentage point regarding readmission with a two-sided significance level of 5\% and a power of $80 \%$. Patients in both the intervention group and the usual practice group will receive standard treatment in relation to their medical conditions; the intervention group will receive the developed intervention.

Alongside the quasi-experimental study, we will make a economic evaluation (aim 2) as a cost-effectiveness analysis with the main parameters being readmission and mortality. A healthcare viewpoint will be taken to estimate the cost of all activities and ressource use related to the patients' rehabilitation. National registers will be used to estimate resource use in primary and secondary healthcare sectors. The cost of the intervention will be based on micro-costing. To assess cost-effectiveness, the incremental cost-effectiveness ratio will be calculated.

Change in performance of daily activities (aim 3) will be examined within the intervention group. Data regarding self-reported limitations in performing daily activities measured with Barthel-20 and WHODAS 2.0 and health-related quality of life measured with EQ-5D will be collected using a structured interview questionnaire during admission and at both 30 days and 26 weeks after discharge [47-50].

Elderly patients' experiences of being discharged from ED and returning to everyday life will be examined in a qualitative study (aim 4). Individual interviews with 10 patients who received the intervention will be conducted. The interviews will be analysed from a phenomenological descriptive viewpoint, using systematic text condensation. Purposive sampling of patients will be used to ensure variety in diagnosis, age, gender, material status and support from primary care, as this could contribute to the richness of data [51]. In accordance with a phenomenological approach, we intend to rely on in-depth and rich data rather than the number of participants and the goal is to achieve data that are detailed, nuanced and of sufficient quality rather than seek data saturation [51].

\section{Discussion}

This paper describes the development and planned evaluation of a complex intervention aimed at reducing the risk of readmission of elderly patients following a stay at the ED. The development followed the six steps in the IM protocol [24].

Using an IM approach in developing interventions has several strengths. Multiple methods, such as interviews, a literature search and involving an expert steering group helped to define the problem and identify methods used to target it. The use of IM during the process ensured that the intervention was systematically described and based on available evidence and theory. The logic model in step 1 enables project planners to be specific about the problem and the underlying determinants and to decide what should change as a result of the intervention.

As on of the goals for the intervention, we chose to focus on enhancing elderly patients' performance of daily activities, as limitations in performing daily activities have been identified as high-risk factors for readmission [1, 3, 7, 16, 17]. Despite the fact that limitations in performing daily activities is documented as a risk factor for readmission, this focus is seldom used in interventions aimed at reducing the risk of readmission in elderly patients. Frequently, interventions that aim to reduce readmission in elderly patients include medical treatment, discharge planning and coordination of care [12, 51-54]. Some studies within comprehensive geriatric care have developed interventions aimed at improving geriatric care patients' performance of daily activities. However, the descriptions of these interventions are insufficient, making it impossible to replicate them $[11,14,15,55]$.

Although IM was found to be a useful and systematic method for describing the intervention, it was also 
time-consuming. Developing matrices with performance objectives (step 2) was particularly time-consuming because of the lack of clear guidance on how to select both the performance objectives and the most important determinants. We chose pragmatically the determinants that had the greatest influence on readmission and were considered changeable and feasible to address in the setting.

In the implementation plan, we chose a different strategy than recommended by the IM protocol. Instead of constructing a matrix with performance objectives and change objectives for the use of the intervention, we found it less time-consuming and more feasible to list the performance objectives.

Patient representatives were not included in the development of the intervention. Involvement of users is generally recognized as important when improving the quality of healthcare services. Lack of user involvement is therefor considered as a limitation in the development of the intervention.

Although it was time-consuming to follow the IM protocol, doing so allowed us to describ and design an intervention that was focused, theory-based and partly evidence-based. Our intention with this paper is to describe how we developed the intervention using different methods, and to describe the intervention with sufficient detail and transparency so that replication is possible. This is in line with the recommendation from the Medical Research Council (MRC) that reporting the underlying theory, methods and strategies is valuable because it enhances the possibility of replicating effective interventions [21].

Further studies will evaluate the effectiveness of the developed intervention and will be conducted according to MRC guidance on how to develop and evaluate complex interventions [21]. This means that besides the systematic development process and pilot testing of the intervention, the evaluation will include an effectiveness evaluation, an economic evaluation and a description of the patients' perspective. The evaluation of effectiveness will contribute with knowledge on strategies for reducing the risk of readmission in elderly patients with limitations in performing daily activities and is an important part of building evidence-based practice in rehabilitation.

\section{Conclusion}

The present paper describes the development and design of a complex intervention using the IM protocol. The intervention is aimed at reducing the risk of readmission of elderly patients discharged from the ED. Despite the time-consuming process, the IM protocol was found to be a useful method with which to guide the development of the complex intervention. It allowed us to develop a theory- and evidence-based intervention that can be delivered in a clinical ED context.

\section{Additional files}

Additional file 1: Keywords and search string. (PDF 27 kb)

Additional file 2: Keywords and search string for effectivenss studies. (PDF 29 kb)

\begin{abstract}
Abbreviations
CGC: Comprehensive geriatric care; ED: Emergency Department; ICF: International classification of functioning, disability and health; IM: Intervention mapping; MoHO: Model of human occupation; MRC: Medical research council; OT: Occupational therapist; PT: Physiotherapist
\end{abstract}

Acknowledgements

We acknowledge participants from the steering group and from the reference group.

Funding

The research was founded by Tryg Foundation, Foundation of Public Health in the Middle and Aase and Ejnar Danielsen's foundation.

Availability of data and materials

All data generated or analysed during this study are included in this published article.

\section{Authors' contributions}

LMN completed the intervention mapping process with feedback from LGOE, conducted the literature review and drafted the manuscript. LGOE, HK, KSP and TM participated in the intervention mapping process and helped to draft the manuscript. All authors read and approved the manuscript.

\section{Ethics approval and consent to participate}

Evaluation of the effectiveness of the intervention is approved by the Danish Data Protection Agency (J.nr. 2012-41-0763) and by the National Health Board (3-3013-608/1/). The regional ethics committee confirmed that approval of the study was not required because biomedical experiments were not included (J. nr.1-10-72-108-14). All patients in the intervention group will receive written and verbal information about the conditions of their participation and sign informed consent prior to inclusion. The study is registered in Clinicaltrial.gov (NCT02078466). The pilot study was approved by the Danish Data Protection Agency (J.nr. 2012-41-0763). All of the participants in the pilotstudy received written and verbal information about the conditions of their participation and signed informed consent prior to inclusion.

Consent for publication

Not applicable.

\section{Competing interests}

The authors declare that they have no competing interests.

\section{Publisher's Note}

Springer Nature remains neutral with regard to jurisdictional claims in published maps and institutional affiliations.

\section{Author details}

${ }^{1}$ Department of Physiotherapy and Occupational Therapy, Aarhus University Hospital, Aarhus, Denmark. ${ }^{2}$ Department of Occupational Therapy, VIA University College, Aarhus, Denmark. ${ }^{3}$ Department of Public Health, Aarhus University, Aarhus, Denmark. ${ }^{4}$ DEFACTUM, Central Denmark Region, Aarhus, Denmark. ${ }^{5}$ Research Center for Emergency Medicine, Aarhus University Hospital, Aarhus, Denmark. 'Department of Health Science and Technolog, Aalborg University, Aalborg, Denmark. ${ }^{7}$ Centre of Research in Rehabilitation (CORIR) Department of Clinical Medicin, Aarhus University and Aarhus University Hospital, Aarhus, Denmark. 
Received: 23 May 2017 Accepted: 15 July 2018

Published online: 28 July 2018

\section{References}

1. Comette P, D'Hoore W, Malhomme B, Van Pee D, Meert P, Swine C. Differential risk factors for early and later hospital readmission of older patients. Aging Clin Exp Res. 2005;17:322-8.

2. Greysen SR, Stijacic Cenzer I, Auerbach AD, Covinsky KE. Functional impairment and hospital readmission in Medicare seniors. JAMA Intern Med. 2015;175:559-65.

3. Chu LW, Pei CK. Risk factors for early emergency hospital readmission in elderly medical patients. Gerontology. 1999;45:220-6.

4. Danish Health and Medicine Authority. Readmission of elderly in Denmark 2008. Copenhagen: Danish Health and Medicine Authority; 2009.

5. McCabe JJ, Kennelly SP. Acute care of older patients in the emergency department: strategies to improve patient outcomes. Open Access Emerg Med. 2015;7:45-54

6. Salvi F, Morichi V, Grilli A, Giorgi R, De Tommaso G, Dessi-Fulgheri P. The elderly in the emergency department: a critical review of problems and solutions. Intern Emerg Med. 2007;2:292-301.

7. Deschodt M, Devriendt E, Sabbe M, Knockaert D, Deboutte P, Boonen S, et al. Characteristics of older adults admitted to the emergency department (ED) and their risk factors for ED readmission based on comprehensive geriatric assessment: a prospective cohort study. BMC Geriatr. 2015;15:54015-0055-7.

8. Aminzadeh F, Dalziel WB. Older adults in the emergency department: a systematic review of patterns of use, adverse outcomes, and effectiveness of interventions. Ann Emerg Med. 2002;39:238-47.

9. Lowthian JA, McGinnes RA, Brand CA, Barker AL, Cameron PA. Discharging older patients from the emergency department effectively: a systematic review and meta-analysis. Age Ageing. 2015;44:761-70.

10. Hastings SN, Heflin MT. A systematic review of interventions to improve outcomes for elders discharged from the emergency department. Acad Emerg Med. 2005;12:978-86.

11. Caplan GA, Williams AJ, Daly B, Abraham K. A randomized, controlled trial of comprehensive geriatric assessment and multidisciplinary intervention after discharge of elderly from the emergency department--the DEED II study. J Am Geriatr Soc. 2004;52:1417-23.

12. Cunliffe AL, Gladman JR, Husbands SL, Miller P, Dewey ME, Harwood RH. Sooner and healthier: a randomised controlled trial and interview study of an early discharge rehabilitation service for older people. Age Ageing. 2004; 33:246-52.

13. Katz EB, Carrier ER, Umscheid CA, Pines JM. Comparative effectiveness of care coordination interventions in the emergency department: a systematic review. Ann Emerg Med. 2012;60:12-23.

14. Legrain S, Tubach F, Bonnet-Zamponi D, Lemaire A, Aquino JP, Paillaud E, et al. A new multimodal geriatric discharge-planning intervention to prevent emergency visits and rehospitalizations of older adults: the optimization of medication in AGEd multicenter randomized controlled trial. J Am Geriatr Soc. 2011:59:2017-28.

15. Mion LC, Palmer RM, Meldon SW, Bass DM, Singer ME, Payne SM, et al. Case finding and referral model for emergency department elders: a randomized clinical trial. Ann Emerg Med. 2003;41:57-68.

16. Campbell SE, Seymour DG, Primrose WR, ACMEPLUS Project. A systematic literature review of factors affecting outcome in older medical patients admitted to hospital. Age Ageing. 2004;33:110-5

17. Caplan GA, Brown A, Croker WD, Doolan J. Risk of admission within 4 weeks of discharge of elderly patients from the emergency department--the DEED study. Discharge of elderly from emergency department. Age Ageing. 1998; 27:697-702.

18. Abizanda P, Leon M, Dominguez-Martin L, Lozano-Berrio V, Romero L, Luengo $C$, et al. Effects of a short-term occupational therapy intervention in an acute geriatric unit. A randomized clinical trial. Maturitas. 2011;69:273-8.

19. Courtney M, Edwards H, Chang A, Parker A, Finlayson K, Hamilton K. Fewer emergency readmissions and better quality of life for older adults at risk of hospital readmission: a randomized controlled trial to determine the effectiveness of a 24-week exercise and telephone follow-up program. J Am Geriatr Soc. 2009;57:395-402.

20. Gitlin LN, Hauck WW, Dennis MP, Winter L, Hodgson N, Schinfeld S. Longterm effect on mortality of a home intervention that reduces functional difficulties in older adults: results from a randomized trial. J Am Geriatr Soc. 2009:57:476-81.

21. Craig P, Dieppe P, Macintyre S, Michie S, Nazareth I, Petticrew M, et al. Developing and evaluating complex interventions: the new Medical Research Council guidance. BMJ. 2008;337:a1655.

22. Hoffmann TC, Glasziou PP, Boutron I, Milne R, Perera R, Moher D, et al. Better reporting of interventions: template for intervention description and replication (TIDieR) checklist and guide. BMJ. 2014;348:g1687.

23. Schulz KF, Altman DG, Moher D, CONSORT Group. CONSORT 2010 statement: updated guidelines for reporting parallel group randomised trials. PLoS Med. 2010;7:e1000251.

24. Eldredge Bartholomew LK, Markham CM, Ruiter RA, et al. Planning health promotion programs, an intervention mapping approach. 4th ed. San Fransico: Wiley; 2016.

25. Salvi F, Mattioli A, Giannini E, Vita D, Morichi V, Fallani M, et al. Pattern of use and presenting complaints of older patients visiting an emergency Department in Italy. Aging Clin Exp Res. 2013;25:583-90.

26. Gill TM, Allore HG, Holford TR, Guo Z. Hospitalization, restricted activity, and the development of disability among older persons. JAMA. 2004;292:2115-24.

27. Gill TM. Assessment of function and disability in longitudinal studies. J Am Geriatr Soc. 2010:58(Suppl 2):S308-12.

28. Pedersen MM, Bodilsen AC, Petersen J, Beyer N, Andersen O, Lawson-Smith $L$, et al. Twenty-four-hour mobility during acute hospitalization in older medical patients. J Gerontol A Biol Sci Med Sci. 2013;68:331-7.

29. Suesada MM, Martins MA, Carvalho CR. Effect of short-term hospitalization on functional capacity in patients not restricted to bed. Am J Phys Med Rehabil. 2007;86:455-62.

30. Zisberg A, Shadmi E, Sinoff G, Gur-Yaish N, Srulovici E, Admi H. Low mobility during hospitalization and functional decline in older adults. J Am Geriatr Soc. 2011;59:266-73.

31. Krevers B, Narvanen AL, Oberg B. Patient evaluation of the care and rehabilitation process in geriatric hospital care. Disabil Rehabil. 2002;24:482-91.

32. Slatyer S, Toye C, Popescu A, Young J, Matthews A, Hill A, et al. Early representation to hospital after discharge from an acute medical unit perspectives of older patients, their family caregivers and health professionals. J Clin Nurs. 2013:22:445-55.

33. World Health Organization. International classification of functioning, disability and Health-ICF. Genève: World Health Organization; 2001.

34. Gladman JR. The international classification of functioning, disability and health and its value to rehabilitation and geriatric medicine. J Chin Med Assoc. 2008;71:275-8.

35. Kielhofner G. Model of human occupation: theory and application. 4th ed. Philadelphia: Lippincott Williams \& Wilkins; 2008.

36. Fisher AG. Occupational therapy intervention process model: three star press, Inc; 2009

37. Petersson I, Lilja M, Hammel J, Kottorp A. Impact of home modification services on ability in everyday life for people ageing with disabilities. J Rehabil Med. 2008;40:253-60

38. Steultjens EM, Dekker J, Bouter LM, Jellema S, Bakker EB, van den Ende $\mathrm{CH}$. Occupational therapy for community dwelling elderly people: a systematic review. Age Ageing. 2004;33:453-60.

39. De Vriendt P, Peersman W, Florus A, Verbeke M, Van de Velde D. Improving health related quality of life and independence in community dwelling frail older adults through a client-Centred and activity-oriented program. A pragmatic randomized controlled trial. J Nutr Health Aging. 2016;20:35-40.

40. Michie S, Richardson M, Johnston M, Abraham C, Francis J, Hardeman W, et al. The behavior change technique taxonomy ( 1 1) of 93 hierarchically clustered techniques: building an international consensus for the reporting of behavior change interventions. Ann Behav Med. 2013;46:81-95.

41. Freiberger $E$, de Vreede $P$, Schoene D, Rydwik E, Mueller V, Frandin K, et al. Performance-based physical function in older community-dwelling persons: a systematic review of instruments. Age Ageing. 2012;41:712-21.

42. Podsiadlo D, Richardson S. The timed "Up \& Go": a test of basic functional mobility for frail elderly persons. J Am Geriatr Soc. 1991;39:142-8.

43. Jones CJ, Rikli RE, Beam WC. A 30-s chair-stand test as a measure of lower body strength in community-residing older adults. Res Q Exerc Sport. 1999;70:113-9.

44. Fisher AG, Bray K. Assessment of motor and process skills. Development, standardization, and administration manual. 7th ed. Colorado: Fort Collins Three Star Press; 2007.

45. Merritt BK. Validity of using the assessment of motor and process skills to determine the need for assistance. Am J Occup Ther. 2011;65:643-50. 
46. Naylor MD, Brooten D, Campbell R, Jacobsen BS, Mezey MD, Pauly MV, et al. Comprehensive discharge planning and home follow-up of hospitalized elders: a randomized clinical trial. JAMA. 1999;281:613-20.

47. Mahoney Fl, Barthel DW. Functional evaluation: the Barthel index. Md State Med J. 1965;14:61-5.

48. Collin C, Wade DT, Davies S, Horne V. The Barthel ADL index: a reliability study. Int Disabil Stud. 1988;10:61-3.

49. Haywood KL, Garratt AM, Fitzpatrick R. Quality of life in older people: a structured review of generic self-assessed health instruments. Qual Life Res. 2005;14:1651-68.

50. Ustun TB, Chatterji S, Kostanjsek N, Rehm J, Kennedy C, Epping-Jordan J, et al. Developing the World Health Organization disability assessment schedule 2.0. Bull World Health Organ. 2010;88:815-23.

51. Fusch PI, Ness LR. Are we there yet? Data saturation in qualitative research. Qual Rep. 2015;20:1408-16.

52. Rosted E, Poulsen I, Hendriksen C, Petersen J, Wagner L. Testing a two step nursing intervention focused on decreasing rehospitalizations and nursing home admission post discharge from acute care. Geriatr Nurs. 2013;34:477-85.

53. Biese K, Lamantia M, Shofer F, McCall B, Roberts E, Stearns SC, et al. A randomized trial exploring the effect of a telephone call follow-up on care plan compliance among older adults discharged home from the emergency department. Acad Emerg Med. 2014;21:188-95.

54. Rytter $L$, Jakobsen HN, Ronholt F, Hammer AV, Andreasen AH, Nissen A, et al. Comprehensive discharge follow-up in patients' homes by GPs and district nurses of elderly patients. A randomized controlled trial. Scand J Prim Health Care. 2010;28:146-53.

55. Torisson G, Minthon L, Stavenow L, Londos E. Multidisciplinary intervention reducing readmissions in medical inpatients: a prospective, non-randomized study. Clin Interv Aging. 2013;8:1295-304.

Ready to submit your research? Choose BMC and benefit from:

- fast, convenient online submission

- thorough peer review by experienced researchers in your field

- rapid publication on acceptance

- support for research data, including large and complex data types

- gold Open Access which fosters wider collaboration and increased citations

- maximum visibility for your research: over $100 \mathrm{M}$ website views per year

At BMC, research is always in progress.

Learn more biomedcentral.com/submissions 